WORKING PAPER · NO. 2020-104

\title{
Tracking Mask Mandates during the COVID-19 Pandemic
}

Austin L. Wright, Geet Chawla, Luke Chen, Anthony Farmer, IPAL Lab, and DPSS Lab AUGUST 2020

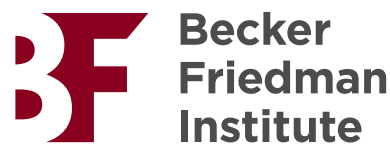




\title{
Tracking Mask Mandates during the COVID-19 Pandemic
}

\author{
Austin L. Wright ${ }^{1}$ Geet Chawla ${ }^{1}$ Luke Chen $^{1}$ Anthony Farmer ${ }^{1}$ \\ IPAL Lab ${ }^{1,2}$ DPSS Lab ${ }^{1}$ \\ ${ }^{1}$ Harris School of Public Policy, University of Chicago \\ ${ }^{2}$ School of Economics, Peking University* \\ First version: July 22, 2020. \\ Updated: August 4, 2020.
}

\begin{abstract}
In this research note, we introduce and describe the collection of data on mask mandates in the United States. We detail the collection protocol as well as tools for accessing the data and offering feedback on amendments to the data. We present introductory visualization of the data. This project is part of a continuing effort to collect vital information about public policy interventions during the COVID-19 pandemic.
\end{abstract}

Data download location: https: / / www . austinlwright.com/covid-research

Recommend an update: https://forms.gle/DtUSyvHDj9rj1Akq8

JEL Classification: H12, I18.

Keywords: COVID-19, mask mandates.

${ }^{*}$ We thank all of the student research assistants in the 2020 IPAL and DPSS labs for their hard work and dedication to this important project. We also thank Julia Raifman and Marcus Painter for their inspiration of this open access data collection and sharing project. All errors remain our own. 


\section{Overview}

In this data overview paper, we introduce a novel comprehensive database of county-level mask mandate policies in the United States. We begin by providing an overview of the current research on mask use during the COVID-19 pandemic. This research suggests that mask use can effectively reduce transmission risk, has significantly reduced cases and deaths in states where relevant policies have been implemented previously, yet compliance and enforcement remains uneven. The first paper using this data, Milosh et al. (2020), highlights the important role of partisanship in influencing mask use. Our goal is to advance research on the public policy relevance of mask mandates by making this data publicly available and free to download and use. Since local policies are constantly evolving, we ask users to contribute to continuous updates of the data. We describe how to suggest revisions. We will update the data frequently and recommend users document their respective retrieval dates.

Figure 1: County-specific and state-wide mask mandates in the United States.

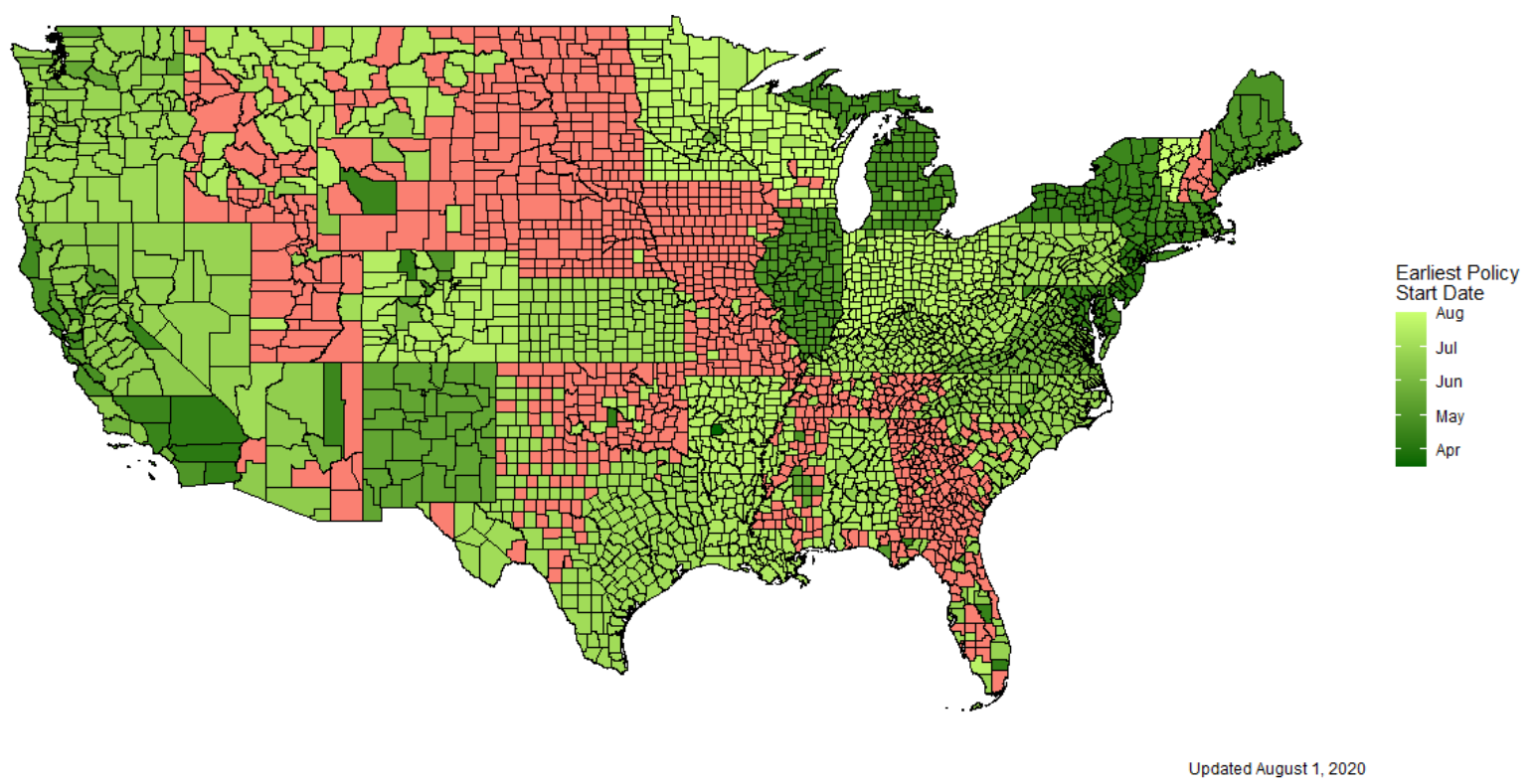

Notes: Data used to compile this map is a combination of county-specific and state-wide mandates assembled as a part of the Tracking Mask Mandates project. The latest data can be retrieved at this link: https://www . austinlwright.com/covid-research. To recommend a revision to the database, please navigate to this link: https://forms.gle/DtUSyvHDj9rj1Akq8. 


\section{Mask Use during the COVID-19 Pandemic}

Usage of masks and fabric face coverings in public spaces has recently received substantial attention as a public health intervention for slowing the spread of COVID-19 within the US. Experimental evidence and mathematical modelling have shown that face mask usage can effectively block droplet transmission, greatly reducing the rate of infection for COVID-19 (Chu et al., 2020; Eikenberry et al., 2020; Fisman et al., 2020; Gandhi et al., 2020; Leung et al., 2020). Additionally, observational studies have demonstrated that mandating mask usage is associated with lower mortality and incidence rates (Cheng et al., 2020; Leffler et al., 2020; Lyu and Wehby, 2020a; Mitze et al., 2020). Most governmental bodies, including the US CDC, have recommended mask usage in public spaces (CDC, 2020). Numerous academic pieces have been published explicitly advocating for increased mask usage (Greenhalgh et al., 2020; Howard et al. 2020; Javid et al., 2020; Kai et al., 2020). However, mask usage in the US remains far from universal; an estimate by the New York Times based on survey results indicates around 80 percent of Americans use masks (Katz et al. 2020). While this figure may appear somewhat high, it would only be the case if this figure applied uniformly to the whole US population.

Identifying patterns in mask usage, or the lack thereof, is useful to assessing the role and effectiveness of mask usage as a public health intervention. Provision of information, enforcement, and other tools for promoting mask usage rely on correctly identifying the determinants and realized effects of mask usage. Some studies have sought to identify correlations between socio-demographic characteristics and mask usage (Fisher et al., 2020; Haischer et al., 2020). These studies found correlations between regions and mask usage but did not account for local mask mandates. If it is the case that mask mandates and regions are correlated - which is revealed to be the case by a map of mask mandates - then the existing analysis may be biased by the omission of mandate data. These studies are emblematic of cases in which local mask mandate data is needed to prevent bias and improve precision. The county-level mask mandate data being provided here has already been utilized by Milosh et al. (2020) in their analyses of the correlation between political partisanship and mask usage to address potential confounding from local mandates.

The provided data could also be used to study the effects of the mask mandates themselves 
on mask usage, COVID-19 incidence, mortality, or other outcomes. Previously, data on stayat-home mandates have been combined with mobility data to identify intention-to-treat effects (Brzezinski et al., 2020; Chernozhukov et al., 2020; Gupta et al., 2020; Lyu and Wehby, 2020b; Wright et al., 2020). With access to county-level mask mandate data, similar analyses could be carried out relatively easily while yielding distinct, useful results. For example, Knotek et al. (2020) attain survey findings that suggest legal enforcement of mask usage is likely to have positive effects on realized mask usage. Instead, findings from a natural experiment are generally preferred to survey results. For example, Betsch et al.(2020) use cross-sectional German data to estimate the effect of mask mandates on mask usage and other behavioral outcomes. Until now, such analyses was not possible in the US context. Such analyses could improve forecasts and potentially help local and state governments make better-informed decisions regarding mask mandates.

\section{Existing Databases}

Raifman et al. (2020) have published a database of state-level policies regarding stay-at-home orders, mask mandates, business closures, housing policies, Medicaid and SNAP policies, and several other topics relevant to Covid-19 response. We thank Raifman et al. (2020) for inspiring this effort. Additionally, some states' county-level policies have been summarised by local media outlets or other organizations. However, no centralized database yet exists containing individualized data on each county in all states. Thus, we are providing this database of countylevel mask mandates to provide researchers with detailed information on mask mandates at the county-level across the US in the hopes that it opens the way to new research questions and improvements to extant research. The data may be used to study the intent-to-treat effects of mask mandates, improve estimate precision or reduce bias in regressions involving mask usage.

\section{Data Collection}

We followed a predefined protocol to collect county-level data on the start and end dates of mask mandate policy in the Unites States. 


\section{Phase I: Baseline data collection and validation by IPAL Lab}

Following is the step-by-step process that we incorporated:

1. Research assistants from the IPAL lab were assigned 5 states of the United States each. Given the size of the program's cohort, more than one research assistant was assigned the same state.

2. The researchers then accumulated the state-level and county-level data on the start date, end date and conditions of the mask mandate policy on their respective states.

3. Conditions of mask mandate policy entailed three choices: all public places, retail only, and unspecified. The choices meant if a mask was required to be worn in all public places, in retail places only, or that the policy was not defined in the county websites (or other credible sources), respectively.

4. In order to cross-validate the aforementioned data points, other researchers who were assigned the same state performed their independent research and verified the data entered by their peers.

5. Mask mandate policy pertaining to only COVID-19 was captured; mask-policies stemming from other reasons or issues (such as pollution) were not captured in the dataset.

6. Other policies relating to COVID-19, like a shelter-in-place were not treated as proxies for a mask-mandate policy. Only if there were a mask mandate, the data was documented.

7. In the dataset, only if there was a state-wide policy the information was recorded in the state section. In case the mask-policy was only implemented by specific counties (and not the whole state), the state data was left blank, and this was captured in the county level data only.

8. If the county's start date of the policy was before its respective State's policy - the county's date was captures in the county-level database and the state wide policy date was captured in the state level database. 
9. In some cases, where the policy was implemented in a city earlier than it was implemented in its respective state or county, the date on which the policy was implemented in the city was treated as the date on which the county implemented its policy. In these specific cases, it was assumed that most population of that county resides in the city which was ahead in policy implementation. It was assumed that most people in the county are likely treated by the city-wide mandate.

10. In case there was no reliable evidence found for counties / states, no data was recorded.

11. In case there was no reliable evidence telling that the policy has been reversed, the enddate was left blank assuming that the policy is still in place.

12. If the researchers found competing evidence for the start date of the policy, the earliest date was recorded in the database.

\section{Phase II: Supplemental data updates and validation by DPSS Lab}

After the first draft of the data was finalized, research assistants in the DPSS lab cross-validated the data again, and checked for updates in state or county level policies. Following steps were incorporated for this process:

1. Research assistants were asked to re-validate the sources already documented and verify the start and end dates of the COVID-19 specific mask mandate policy. The conditions of the policy were also re-validated by the researchers.

2. Every researcher was assigned one state to check for a statewide mandate. Additionally, each of them were also assigned 67 counties to check for county-wide mask mandates.

After an intermediate version of data was procured, the authors performed spot-checks to finalize the data that is now publicly available for public use. 


\section{Data Access and Revisions}

\section{Access}

We are making the data publicly available for download at this location. Navigate to the Tracking Mask Mandates section and click the latest link. It will take you to a database hosted as a Google Sheet, which is downloadable. The date of the last update will be included in the title of the sheet.

Link: https://www.austinlwright.com/covid-research.

Data Collection: Tracking Mask Mandates

During summer 2020, I led two research labs that collected a comprehensive, county-specific database of local mask mandates. To advance scientific research on the importance, phased roll out, and downstream consequences of mask mandates, we have made this data publicly available and free to download. We ask anyone using the data to cite our working paper and acknowledge the source of the data.

Figure 2: Website interface for downloading latest Tracking Mask Mandates database. 


\section{Recommending a revision}

We strongly encourage users to evaluate the data retrieved and offer feedback for the database. To formalize this process, we have launched a Google Form that we can use to track and implement recommended amendments to the database.

\section{Link: https:// forms.gle/DtUSyvHDj9rj1Akq8}

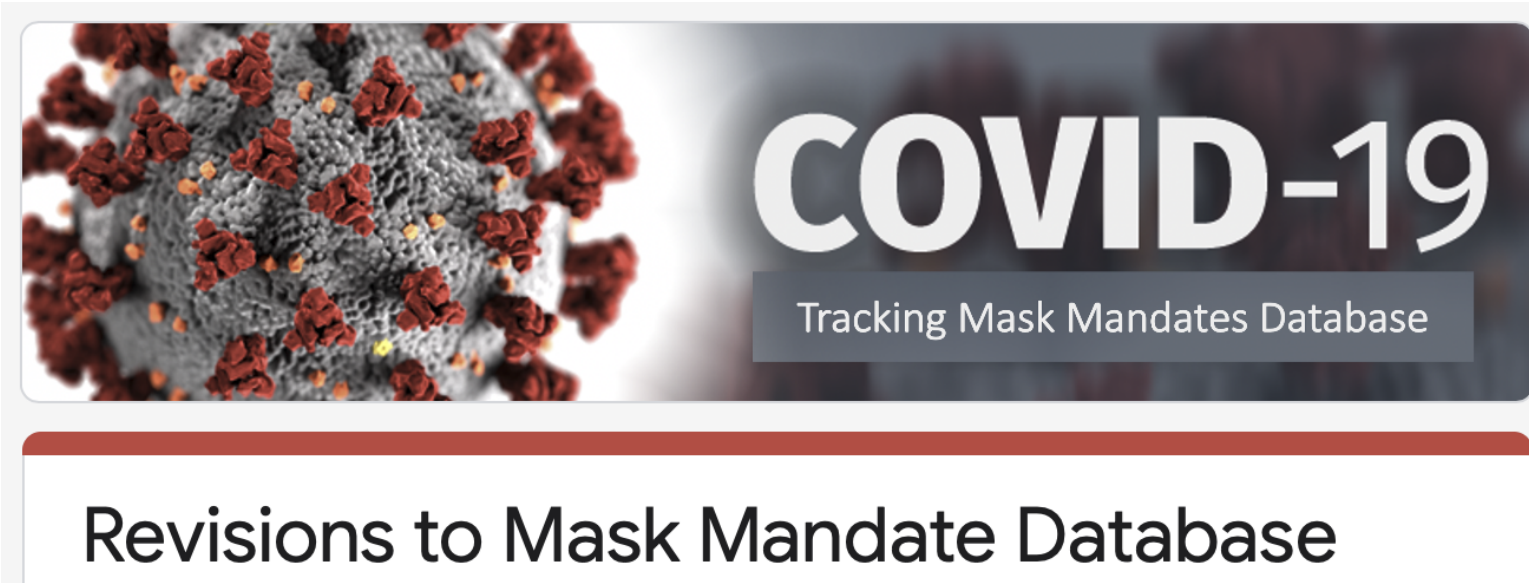

This form is for use for recommended amendments to the The University of Chicago's Tracking Mask Mandates project. If you find an error or would an update that has not yet been made to the database, please use this form.

Citation: Tracking Mask Mandates during the COVID-19 Pandemic. Austin L. Wright, Geet Chawla, Luke Chen, Anthony Farmer, IPAL Lab, DPSS Lab. 2020.

* Required

Notes on Recommended Revision *

Your answer

Figure 3: Google Form interface for recommending revisions to the Tracking Mask Mandates database. 


\section{References}

Cornelia Betsch, Lars Korn, Philipp Sprengholz, Lisa Felgendreff, Sarah Eitze, Philipp Schmid, and Robert Böhm. Social and behavioral consequences of mask policies during the COVID19 pandemic, 2020. URL https://osf.io/gn6c9.

Adam Brzezinski, Valentin Kecht, David Van Dijcke, and Austin L. Wright. Belief in science influences physical distancing in response to COVID-19 lockdown policies, 2020. URL https://papers.ssrn.com/abstract $=3587990$.

CDC. Coronavirus disease 2019 (COVID-19), 2020. URL https:// Www.cdc.gov/coronavirus/2019-ncov/prevent-getting-sick/ cloth-face-cover-guidance.html. Library Catalog: www.cdc.gov.

Vincent Chi-Chung Cheng, Shuk-Ching Wong, Vivien Wai-Man Chuang, Simon Yung-Chun So, Jonathan Hon-Kwan Chen, Siddharth Sridhar, Kelvin Kai-Wang To, Jasper Fuk-Woo Chan, Ivan Fan-Ngai Hung, Pak-Leung Ho, and Kwok-Yung Yuen. The role of communitywide wearing of face mask for control of coronavirus disease 2019 (COVID-19) epidemic due to SARS-CoV-2. 81(1):107-114, 07 2020. ISSN 0163-4453. doi: 10.1016/j.jinf.2020.04. 024. URL/https://www.ncbi.nlm.nih.gov/pmc/articles/PMC7177146/,

Victor Chernozhukov, Hiroyuki Kasahara, and Paul Schrimpf. Causal impact of masks, policies, behavior on early covid-19 pandemic in the u.s. page 2020.05.27.20115139, 2020. doi: 10.1101/2020.05.27.20115139. URL https://www.medrxiv.org/content/10. $1101 / 2020.05 .27 .20115139 v 5$. Publisher: Cold Spring Harbor Laboratory Press.

Derek K. Chu, Elie A. Akl, Stephanie Duda, Karla Solo, Sally Yaacoub, Holger J. Schünemann, Elie A. Akl, Amena El-harakeh, Antonio Bognanni, Tamara Lotfi, Mark Loeb, Anisa Hajizadeh, Anna Bak, Ariel Izcovich, Carlos A. Cuello-Garcia, Chen Chen, David J. Harris, Ewa Borowiack, Fatimah Chamseddine, Finn Schünemann, Gian Paolo Morgano, Giovanna E. U. Muti Schünemann, Guang Chen, Hong Zhao, Ignacio Neumann, Jeffrey Chan, Joanne Khabsa, Layal Hneiny, Leila Harrison, Maureen Smith, Nesrine Rizk, Paolo Giorgi Rossi, Pierre AbiHanna, Rayane El-khoury, Rosa Stalteri, Tejan Baldeh, Thomas Piggott, 
Yuan Zhang, Zahra Saad, Assem Khamis, Marge Reinap, Stephanie Duda, Karla Solo, Sally Yaacoub, and Holger J. Schünemann. Physical distancing, face masks, and eye protection to prevent person-to-person transmission of SARS-CoV-2 and COVID-19: a systematic review and meta-analysis. 395(10242):1973-1987, 2020. ISSN 0140-6736, 1474547X. doi: 10.1016/S0140-6736(20)31142-9. URL https://doi .org/10.1016/ S0140-6736 (20) 31142-9. Publisher: Elsevier.

Steffen E. Eikenberry, Marina Mancuso, Enahoro Iboi, Tin Phan, Keenan Eikenberry, Yang Kuang, Eric Kostelich, and Abba B. Gumel. To mask or not to mask: Modeling the potential for face mask use by the general public to curtail the COVID-19 pandemic. 5: 293-308, 2020. ISSN 2468-0427. doi: 10.1016/j.idm.2020.04.001. URL http: / /www . sciencedirect.com/science/article/pii/s2468042720300117.

Kiva A. Fisher, John P. Barile, Rebecca J. Guerin, Kayla L. Vanden Esschert, Alexiss Jeffers, Lin H. Tian, Amanda Garcia-Williams, Brian Gurbaxani, William W. Thompson, and Christine E. Prue. Factors associated with cloth face covering use among adults during the COVID19 pandemic - united states, april and may 2020. 69(28):933-937, 2020. ISSN 0149-2195, 1545-861X. doi: 10.15585/mmwr.mm6928e3. URL http://www.cdc.gov/mmwr/ volumes/69/wr/mm6928e3.htm?s_cid=mm6928e3_w.

David N. Fisman, Amy L. Greer, and Ashleigh R. Tuite. Bidirectional impact of imperfect mask use on reproduction number of COVID-19: A next generation matrix approach. 5:405-408, 2020. ISSN 2468-2152. doi: 10.1016/j.idm.2020.06.004. URL https://www .ncbi . nlm.nih.gov/pmc/articles/PMC7334658/,

Monica Gandhi, Chris Beyrer, and Eric Goosby. Masks do more than protect others during COVID-19: Reducing the inoculum of SARS-CoV-2 to protect the wearer. 2020. ISSN 1525-1497. doi: 10.1007/s11606-020-06067-8. URL https://doi .org/10 .1007/ s11606-020-06067-8.

Trisha Greenhalgh, Manuel B Schmid, Thomas Czypionka, Dirk Bassler, and Laurence Gruer. Face masks for the public during the covid-19 crisis. page m1435, 2020. ISSN 1756- 
1833. doi: 10.1136/bmj.m1435. URL http://wWw.bmj.com/lookup/doi/10 . $1136 / \mathrm{bmj} \cdot \mathrm{m1435}$.

Sumedha Gupta, Thuy D Nguyen, Felipe Lozano Rojas, Shyam Raman, Byungkyu Lee, Ana

Bento, Kosali I Simon, and Coady Wing. Tracking public and private responses to the COVID-19 epidemic: Evidence from state and local government actions, 04 2020. URL http: / /www.nber.org/papers/w27027. Series: Working Paper Series.

Michael H Haischer, Rachel Beilfuss, Meggie Rose Hart, Lauren Opielinski, David Wrucke, Gretchen Zirgaitis, Toni D Uhrich, and Sandra K Hunter. Who is wearing a mask? gender-, age-, and location-related differences during the COVID19 pandemic. page 2020.07.13.20152736, 2020. doi: 10.1101/2020.07.13. 20152736. URL http://medrxiv.org/content/early/2020/07/20/2020. 07.13 .20152736$. abstract.

Jeremy Howard, Austin Huang, Zhiyuan Li, Zeynep Tufekci, Vladimir Zdimal, Helene-Mari van der Westhuizen, Arne von Delft, Amy Price, Lex Fridman, Lei-Han Tang, Viola Tang, Gregory L. Watson, Christina E. Bax, Reshama Shaikh, Frederik Questier, Danny Hernandez, Larry F. Chu, Christina M. Ramirez, and Anne W. Rimoin. Face masks against COVID19: An evidence review. 2020. doi: 10.20944/preprints202004.0203.v1. URL https: //wWw.preprints.org/manuscript/202004.0203/v1. Publisher: Preprints.

Babak Javid, Michael P Weekes, and Nicholas J Matheson. Covid-19: should the public wear face masks? page m1442, 2020. ISSN 1756-1833. doi: 10.1136/bmj.m1442. URL http: //wWw.bmj.com/lookup/doi/10.1136/bmj.m1442.

De Kai, Guy-Philippe Goldstein, Alexey Morgunov, Vishal Nangalia, and Anna Rotkirch. Universal masking is urgent in the COVID-19 pandemic: SEIR and agent based models, empirical validation, policy recommendations. 2020. URL http://arxiv.org/abs/2004 . 13553.

Josh Katz, Margot Sanger-Katz, and Kevin Quealy. A detailed map of who is wearing masks in 
the u.s. 2020. ISSN 0362-4331. URL https://www.nytimes.com/interactive/ 2020/07/17/upshot/coronavirus-face-mask-map.html.

Edward S. Knotek, Raphael S. Schoenle, Alexander M. Dietrich, Gernot J. Müller, Kristian Ove R. Myrseth, and Michael Weber. Consumers and COVID-19: Survey results on mask-wearing behaviors and beliefs. pages 1-7, 2020. ISSN 2163-3738, 0428-1276. doi: 10.26509/frbc-ec-202020. URL https://clevelandfed.org/newsroom-and-events/ publications/economic-commentary/2020-economic-commentaries/ ec-202020-survey-results-on-mask-wearing-behaviors-and-beliefs. aspx.

Christopher T. Leffler, Edsel B. Ing, Joseph D. Lykins, Matthew C. Hogan, Craig A. McKeown, and Andrzej Grzybowski. Association of country-wide coronavirus mortality with demographics, testing, lockdowns, and public wearing of masks. page 2020.05.22.20109231, 2020. doi: 10.1101/2020.05.22.20109231. URL https://www.medrxiv.org/ content/10.1101/2020.05.22.20109231v4. Publisher: Cold Spring Harbor Laboratory Press.

Nancy H. L. Leung, Daniel K. W. Chu, Eunice Y. C. Shiu, Kwok-Hung Chan, James J. McDevitt, Benien J. P. Hau, Hui-Ling Yen, Yuguo Li, Dennis K. M. Ip, J. S. Malik Peiris, WingHong Seto, Gabriel M. Leung, Donald K. Milton, and Benjamin J. Cowling. Respiratory virus shedding in exhaled breath and efficacy of face masks. 26(5):676-680, 2020. ISSN 1546-170X. doi: 10.1038/s41591-020-0843-2. URL https://doi .org/10.1038/ s41591-020-0843-2.

Wei Lyu and George L. Wehby. Community use of face masks and COVID-19: Evidence from a natural experiment of state mandates in the US. page 10.1377/hlthaff.2020.00818, 2020a. ISSN 0278-2715. doi: 10.1377/hlthaff.2020.00818. URL https://www . healthaffairs.org/doi/full/10.1377/hlthaff.2020.00818. Publisher: Health Affairs. 
Wei Lyu and George L. Wehby. Shelter-in-place orders reduced COVID-19 mortality and reduced the rate of growth in hospitalizations. page 10.1377/hlthaff.2020.00719, 2020b. ISSN 0278-2715. doi: 10.1377/hlthaff.2020.00719. URL https://www . healthaffairs . org/doi/full/10.1377/hlthaff.2020.00719. Publisher: Health Affairs.

Maria Milosh, Marcus Painter, David Van Dijcke, and Austin L. Wright. Unmasking partisanship: How polarization influences public responses to collective risk, 2020. URL https: / / papers.ssrn.com/abstract $=3664779$.

Timo Mitze, Reinhold Kosfeld, Johannes Rode, and Walde Klaus. Face masks considerably reduce COVID-19 cases in germany: A synthetic control method approach. (13319), 2020. URL https://www.iza.org/publications/dp/13319/ face-masks-considerably-reduce-covid-19-cases-in-germany-a-synthetic-con

Julia Raifman, Kristen Nocka, David Jones, Jacob Bor, Sarah Lipson, Jonathan Jay, Megan Cole, Noa Krawczyk, Philip Chan, and Sandro Galea. COVID-19 US state policy database. 2020. doi: 10.3886/E119446V18. URL https://wWw.openicpsr.org/ openicpsr/project/119446/version/V18/view, Publisher: ICPSR - Interuniversity Consortium for Political and Social Research Version Number: 18.

Austin L. Wright, Konstantin Sonin, Jesse Driscoll, and Jarnickae Wilson. Poverty and economic dislocation reduce compliance with COVID-19 shelter-in-place protocols, 2020. URL https://papers.ssrn.com/abstract $=3573637$. 